\title{
Nochmals zur Frage der Unterscheidbarkeit rechts- und linksäugiger Eindrücke.
}

Von

\author{
Dr. A. Brïckner und Dr. E. Th. von Brücke.
}

(Mit 1 Textfigur.)

Wenn wir uns dazu entschliessen, im folgenden noch einmal auf die Frage der Unterscheidbarkeit der Eindrücke beider Augen zurückzukommen, so geschieht es aus verschiedenen Gründen.

In erster Linie war für uns der Umstand massgebend, dass wir in unseren früheren Arbeiten ${ }^{1}$ ) nicht in der Lage waren, auf die Untersuchungen von Bourdon ${ }^{2}$ ) einzugehen, welche $\mathrm{He}$ in $e^{3}$ ) nur kurz referiert hatte, und von denen uns auch nur ein sehr knappes Resümee in der Zeitschrift für Psychologie und Physiologie der Sinnesorgane ${ }^{4}$ ) bekannt geworden war. Erst kürzlich wurden wir durch die Liebenswürdigkeit des Herrn Verfassers in den Besitz seiner ersten Arbeit $^{5}$ ) gesetzt, deren Inhalt uns zusammen mit demjenigen eines späteren Aufsatzes desselben Autors ${ }^{6}$ ) zu einer Nachprüfung der mitgeteilten Versuche veranlasste, worüber hier kurz berichtet werden soll. Ein zweiter Grund lag in der Absicht, einzelne An-

1) Brückner und v. Brücke, Zur Frage der Unterscheidbarkeit rechtsund linksäugiger Gesichtseindrücke. Pflüger's Archiv Bd. 90 S. 290-302, und v. Brücke und Brückner, Über ein scheinbares Organgefühl des Auges. Pflüger's Archiv Bd. 91 S. 360-372.

2) Bourdon, La distinction locale des sensations correspondantes des denx yeux. Bulletin de la société scientifique et médicale de l'ouest. Neuvième année 1900. T. 9 No. 1. Rennes.

3) Heine, Die Unterscheidbarkeit rechtsäugiger und linksäugiger Wahrnehmungen. Klin. Monatsbl. für Augenheilkunde Bd. 39 (2) S. 615-620. 1900.

4) Referat der zitierten Arbeit von Bourdon. Zeitschr. f. Psychol. u. Physiol. der Sinnesorgane Bd. 27 S. 119.

5) Bourdon, 1. c.

6) Bourdon, Sur la distinction des sensations des deux yeux. L'année psychologique. Neuvième année 1903 p. $41-56$. 
gaben in der Literatur, welche uns erst nachträglich bekannt geworden sind, wenigstens kurz zu zitieren sowie einen neuen Versuch mitzuteilen. Drittens endlich sehen wir uns mit zu den folgenden Ausführungen veranlasst durch eine Entgegnung Heine's ${ }^{1}$ ) auf unsere früheren Mitteilungen, in welcher einige Punkte uns der sachlichen Aufklärung zu bedürfen scheinen.

Von älteren Untersuchungen, welche sich auf unsere Frage beziehen, möchten wir die Arbeiten von $\mathrm{Schön}^{2}$ ) nicht unerwähnt lassen ${ }^{3}$ ), welche auf das binokulare Sehen Bezug haben. Im Verlaufe seiner Untersuchungen hat er auch Experimente angestellt, in welchen er nur monokulare oder einseitig stärkere Belichtungen mittelst elektrischer Funken vornahm. Einzelne seiner Versuchspersonen waren in der Lage, anzugeben, welches Auge allein bezw. stärker belichtet wurde. Über die Angaben der Versuchspersonen, wodurch sie zu der Unterscheidung befähigt würden, schreibt Schön,

„dass sie aus der Lage des Punktes nichts hätten entnehmen können, da derselbe bald dem einen bald dem anderen Auge an derselben Stelle erschienen sei, sie hätten dagegen ein Gefühl im betreffenden Auge, dass dieses stärker beleuchtet werde als das andere. Eine genauere Charakterisierung dieses Gefühls konnte ich bisher nicht erlangen. $\left.{ }^{4}\right)^{4}$

Durch Herrn Hofrat S. Exiner wurden wir ferner auf eine Abhandlung von Fleischl${ }^{5}$ ) hingewiesen, welcher ebenfalls hierhergehörige Versuche angestellt hat und das Ergebnis seiner Untersuchungen in folgenden Worten zusammenfasst:

„Hierbei zeigte es sich, dass mein Urteil ungefähr so oft falsch war, als es falsch sein musste unter der Voraussetzung, dass wir keine Kenntnis davon haben, mit welchem unserer beiden Augen wir etwas sehen; ... so dass ich mich auch für berechtigt halte, den Satz anszusprechen, dass wir keine unmittelbare Kenntnis davon haben, ob wir monoculär oder binoculär sehen.“ . . „A Aus diesen Ver-

1) Heine, Zur Frage der Unterscheidbarkeit rechts- und linksäugiger Gesichtseindrücke. Pflüger's Arch. Bd. 101 S. 67-70.

2) Schön, Zur Lehre v. binokularen Sehen. Graefe's Arch: f. Ophthalmologie Bd. 22 (4) S. 31-62; Zur Lehre vom binokularen Sehen, ebenda Bd. 24 (1) S. 27-130; Zur Lehre vom binokularen Sehen, ebenda Bd. 24 (4) S. 47-116.

3) Auf dieselben sowie anf die oben folgende Notiz bei Javal wurden wir durch die Bourdon'schen Arbeiten aufmerksam gemacht.

4) Graefe's Arch. Bd. 24 (4) S. $63 \mathrm{f}$.

5) Fleischl v. Marxow, Gesammeite Abhandlnngen. Physiologischoptische Notizen I S. 149-151. 
Nochm. zur Frage d. Unterscheidbarkeit rechts- u. linksäugiger Eindrücke. 265

suchen scheint mir hervorzugehen, dass ein Organgefühl der Augen in dem Sinne, wie es in neverer Zeit gelegentlich zur Beantwortung physiologischer und psychologischer Fragen angenommen wurde, nicht existiert. Es mag immerhin zngegeben werden, dass sehr bald nach excessiven adäquaten Sinnesreizen sich Veränderungen in dem gereizten Organe sekundär ausbilden, die nun vermittelst der dem Organe angehörigen schmerzempfindenden Nerven wahrgenommen und richtig Iokalisiert werden - eine direkte Wahrnehmung der anatomischen Lage des gereizten Sinnesapparates, ein Organgefühl, kann selbst bei sehr intensiven Reizen - für das Auge wenigstens - nach dem eben Mitgetheilten nicht zugegeben werden."

\section{Auch folgende Mitteilung Javal's ${ }^{1}$ ) scheint uns der Erwähnung} wert zu sein. Fr sagt:

"Quand un objet éloigné se présente aux deux yeux sous deux aspects absolument identiques, il serait sans aucune utilité d'avoir la notion de deux sensations; c'est pourquoi nous voyons, sans chercher quelle part revient à chaque œil. Au contraire, dès que nous avons intérêt à établir cette distinction, nous y parvenons plus ou moins facilement. C'est ainsi que les personnes qui portent constamment des lunettes reconnaissent souvent sur lequel des verres se trouve une tache qui vient à leur obscurcir la vue: c'est là un retour à l'individualité native des sensations des deux yeux ... C'est ainsi enfin qu'une de mes petites strabiques très intelligente, à qui des exercices venaient de restituer la vision binoculaire savait parfaitement dire à laquelle des deux images appartenaient les taches que j'avais faites sur une photographie avant de la lai présenter dans le stéréoscope: elle possédait encore la notion de l'individualité des deux yeux, malgré la fusion des deux images: il y avait distinction consciente des sensations des deux yeux. Quand je lú demandais comment elle reconnaissait à quel ceil appartenait la sensation, elle répondait: je le sens bien."

Im Anschluss an diese zuletzt erwähnte Beobachtung Javal's sei nochmals auf die ähnliche Mitteilung bei Helmholtz ${ }^{2}$ ) hingewiesen, welche wir bereits an anderer Stelle ${ }^{3}$ ) zitiert haben.

Die Arbeiten von Bourdon möehten wir etwas eingehender referieren, da sie schwerer zugänglich sind. Für die vorliegende Frage sind sie aber schon aus dem Grunde von Bedeutung, weil Bourdon als erster in letzter Zeit diesem Problem wieder seine Aufmerksamkeit zugewendet hat.

In der älteren Arbeit ${ }^{4}$ ), welche Heine zum Ausgangspunkt seiner Untersuchungen ${ }^{5}$ ) genommen hatte, berichtet Bourdon über

1) Javal, Manuel du Strabisme p. 23. Paris 1896.

2) Helmboltz, Handbuch der physiologischen Optik. 2. Aufl. S. 894.

3) Pflüger's Arch. Bd. 90 S. 300 und Bd. 91 s. 361.

4) Bullet. de la soc. scient. et méd. de l'ouest 1900 p. $1 \mathrm{ff}$.

5) Klin. Monatsbl. f. Augenheilk. Jahrg. $39 \mathrm{Bd} .2$ S. $615 \mathrm{ff}$. 
eine Reihe von Versuchen ${ }^{1}$ ), welche sich in $\mathrm{zwei}$ verschiedene Gruppen ordnen lassen. Bei denjenigen der ersten Gruppe findet für ein Auge die Verdeckung eines kleinen leuchtenden Punktes im Dunkelraume statt, nachdem vorher beide Augen denselben gesehen hatten. In der anderen Gruppe ist bereits beim Beginne der Beobachtung der leuchtende Punkt für ein Auge unsichtbar.

Die Verdeckung des Punktes wurde bei den Versuchen der ersten Gruppe durch zwei rechteckige Flügel (s. Figur) ${ }^{2}$ ) erreicht, welche auf einer horizontalen Achse $A B$, die parallel zur Frontalebene des Beobachters verlief, befestigt waren. Bei der langsamen Rotation der Achse wurde bald das rechte oder das linke Auge des Beobachters verdeckt, bald waren beide

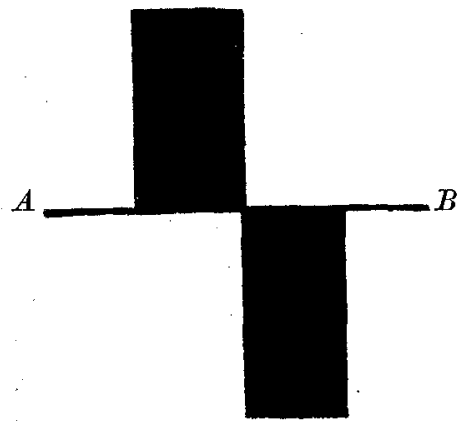
Augen freigegeben. Die Verdeckung des leuchtenden Punktes erfolgte stets von unten her.

Bourdon beobachtete nun sowohl, wenn er den leuchtenden Punkt ${ }^{3}$ ) direkt, als auch wenn er ihn indirekt betrachtete (in letzterem Falle diente ein ständig binokular sichtbarer zweiter leuchtender, horizontal neben dem ersten befindlicher Punkt als Fixierobjekt), dass im Moment der Verdeckung für das eine Auge eine Art Schatten auftauchte, „qui est nettement situé du même côté que l'œil qui ne voit plus l'objet et qui cesse, quand revient la vision binoculaire, du côté de l'autre oeil" ${ }^{4}$ ). Bei einer anderen Versuchsreihe befanden sich die beiden leuchtenden Punkte übereinander; der untere wurde fixiert, der obere für das eine Auge verdeckt.

In diesem Falle erschien der beschriebene Schatten nun nicht auf derjenigen Seite des leuchtenden Punktes, welche dem verdeckten Auge entsprach, sondern auf der anderen.

Auf Grund dieses „objektiven Phänomens", wie Bourdon es

1) Dieselben sind nach des Autors eigener Angabe ohne wesentliche Änderungen auch in seinem Werke "La perception visuelle de l'espace" (p. 225-233) 1902 wiedergegeben.

2) Die Abbildung ist der Arbeit Bourdon's entlehnt.

3) Auch vertikale leuchtende Linien wurden verwendet.

4) 1. c. S. 11 . 
Nochm. 'zur Frage d. Unterscheidbarkeit rechts- u. linksäugiger Eindrücke. 267

an anderer Stelle ${ }^{1}$ ) nennt, war nun für ihn unter den angegebenen Bedingungen fast stets bei den unwissentlichen Versuchen, welche er anstellte, das richtige Urteil möglich, welches Auge das allein sehende war.

In der zweiten Gruppe von Versuchen, welche darin bestanden, dass ein Auge bereits von vornherein ganz oder teilweise vom Sehakte ausgeschlossen war, waren die Versuchsbedingungen ähnlich. Bourdon liess die oben beschriebenen Flügel sich drehen, während er die Augen schloss. Darauf arretierte er das Uhrwerk, welches die Flügel in Bewegung setzte, und stellte sich nun die Aufgabe, anzugeben, ob er binokular oder monokular sah, und, wenn letzteres der Fall war, welches Auge allein sah. Er machte analoge Versuche, wie soeben für die erste Methode beschrieben wurde: zunächst verwendete er einen einzigen leuchtenden Punkt, dann zwei horizontal nebeneinander befindliche Punkte, von denen der eine, indirekt gesehene für das eine Auge unsichtbar war. Im letzten Falle wurde entweder die Fixation des binokular gesehenen Punktes streng festgehalten oder der Blick von einem Punkt zum anderen hin und her bewegt. Endlich wurden statt eines dauernd binokular sichtbaren Punktes deren dreizehn verwendet, welche in Form eines Quadrates angeordnet waren, dessen Seiten aus je drei und dessen Diagonalen aus je fünf Punkten bestanden.

In allen diesen Fällen war das richtige Urteil fast ausnahmslos möglich, wenn auch im letzten Falle nur mit Schwierigkeit, und zwar gelang die Entscheidung auf Grund eines „subjektiven Phänomens", wie der Verfasser die eigenartige Sensation, welche er beobachtete, an anderer Stelle ${ }^{2}$ ) bezeichnet hat. Er gibt folgende Beschreibung von dieser Empfindung:

„... j'étais arrivé à reconnaître à la fois quel oeil était impressionné et quel ceil ne l'était pas; mais, au début, je reconnaissais plutôt avec quel oeil je ne voyais pas; je le reconnaissais à une sorte de gêne et d'engourdissement léger qui se faisait sentir dans cet oeil. Plus tard, j'ai remarqué inversement une sensation de facilité dans l'œil qui voyait, et je reconnaissais également qu'il y avait vision binoculaire par une sensation générale de facilité dans tout l'appareil visuel." )

1) L'année psychologique 1903 p. 43 ff.

2) Ebenda 1903 p. $47 \mathrm{ff}$.

3) Bullet. de la soc. scient. et méd. de l'onest 1900 p. 12. 


\section{An anderer Stelle findet sich die Bemerkung:}

"Les sensations sont toujours celles d'engourdissement et de fatigue dans l'œil qui ne voit pas, de facilité au contraire dans celui qui voit; le clignement des yeux m'aide à les constater." 1 )

Bourd on gelangt nun auf Grund der vorstehend referierten Versuche am Schlusse seines ersten Aufsatzes zu folgender theoretischen Deutung der beiden von ihm beobachteten Phänomene. In bezug auf das "objektive" Phänomen - den unter gewissen Versuchsbedingungen rechts oder links vom Punkte auftauchenden Schatten - sagt er folgendes ${ }^{2}$ ):

„Si on considère les deux rétines, les sensations fournies par les points ,identiques ${ }^{\imath}$ ne sont pas identiques quant au signe local; un point de la rétine droite, comparé au point identique de la rétine gauche, confère à l'impression qu'il recoit le signe local à droite, et réciproquement. Toutefois la différence de position apparente d'un point qui peut résulter du fait que ce point est perçu par la rétine droite ou par le point identique de la rétine gauche est peu considerable. " ${ }^{3}$ )

Das von ihm beobachtete Gefühl der Vertaubung, der Ermüdung im nicht oder weniger sehenden Auge, sowie das Gefühl der Leichtigkeit im anderen fasst Bourdon auf als ein Muskelgefühl. Er sagt $\left.{ }^{4}\right)$ :

„Quant aux appareils musculaires de chacun des yeux, ils différent également l'un de l'autre au point de vue de signe local. Les muscles de l'œil droit donnent, comparativement à ceux de l'œil gauche, la sensation subjective d'à droite, et inversement. Même lorsque les deux yeux fixent un point permanent et par conséquent gardent constamment la même position, l'inégale excitation des denx rétines provoque dans les appareils musculaires des deux yeux des sensations différentes qui sont assez facilement reconnues comme sensations soit de l'œil gauche, soit de l'œil droit .... . Ces sensations musculaires qui se localisent indubitablement soit à droite, soit à gauche suivant quelles proviennent de l'œil drott ou de l'œil gauche suffiraient à elles seules, quand même les sensations des points identiques des rétiffes seraient elles-mêmes primitivement identiques, à différencier ces dernières peu à peu; toute excitation rétinienne de l'œil droit, par' exemple, s'associe en effet à une sensation musculaire de facilité dans cet œil."

1) Bullet. de la soc. scient. et méd. de l'ouest 1900 p. 16.

2) Ebenda p. 19.

3) Heine gelangte auf Grund seiner Versuche, die sich aber mehr den Bourd on'schen Versuchen nähern, welche oben als zweite Gruppe zusammengefasst sind, zu einer ähnlichen theoretischen Auffassung. (Klin. Monatsbl. $\stackrel{f}{\text { L. }}$ Augenheilk. 1. $\left.c_{\text {co }}\right)$

4) 1. c. p. 19 . 
Nochm. zur Frage d. Unterşcheidbarkeit rechts- u. linksäugiger Eindrücke. 269

In seiner zweiten Arbeit ${ }^{1}$ ) macht sich Bourdon nun selbst den Einwand, dass das „objektive Phänomen" vielleicht auf einer übermässigen Konvergenz der Augen im Dunkelraume beruhen könne, wodurch ein (teilweiser) Zerfall des leuchtenden Punktes (bezw. der Linie) in gleichnamige Doppelbilder eintreten könnte. In diesem Falle wäre es erklärt, warum der Schatten stets auf der dem verdeckten Auge entsprechenden Seite auftritt. Bourdon entkräftet diesen Einwand durch eine Anzahl von Versuchen, auf welche wir hier nicht näher eingehen wollen.

Er teilt dann noch eine Reihe von Experimenten mit, welche mittelst des Stereoskops angestellt wurden, in das eine schwarze Tafel mit zwei Öffnungen eingesetzt wurde, welche bei den verschiedenen Versuchen 0,5 oder $2 \mathrm{~mm}$. Durchmesser hatten. Nach der binokularen Verschmelzung derselben wurde der durch die Öffnungen sichtbare - bald weisse, bald farbige oder schwarze - Grund mittelst rotierender farbiger oder weisser Flügel für das eine Auge durch eine andere Farbe verdeckt. Anstatt also dem einen Auge das Sehobjekt ganz zu entziehen, wie bei den Versuchen im Dunkelzimmer, wurde hier statt des ersten Eindrucks dem einen Auge ein andersfarbiger geboten. Die diesbezüglichen Versuche im einzelnen zu referieren, dürfte zu weit führen. Es seien darum nụr kurz die von Bourdon gewonnenen Resultate mitgeteilt:

Auch hier liess sich eine Art Schatten beobachten, wenn der durch die Öffnungen gesehene helle Grund durch eine dunklere Farbe für das eine Auge ersetzt wurde; je nach dem Helligkeitsverhältnis war derselbe deutlicher oder weniger deutlich. Bei etwa gleicher Helligkeit $\mathrm{zwischen}$ substituierter und substituierender Farbe fehlte die Erscheinung, welche Bourdon mit der oben als „objektives Phänomen" bezeichneten identifiziert. Ausserdem konnte mehr oder weniger deutlich auch noch das "subjektive Phänomen" beobachtet werden. Bourdon hat ferner besonders bei diesen : Versuchen mehrfach den Eindruck gewonnen, dass objektives und subjektives Phänomen eng zusammenhängen, ja, vielleicht ein und dasselbe Phänomen sind. : Er sagt darüber folgendes:

"La conclusion qui se dégage pour moi de ces dernières expériences est que le phénomène objectif est lié étroitement au phénomène subjectif, ... q qu'il sē manifeste ou cesse de se manifester en même temps que lui, qu'il ne se constate,

1) L'année psychologique 1903 p. $48 \mathrm{ff}$ 
dans les cas qui ont été précédemment étudiěs, que quand les couleurs vues par les deux yeux diffèrent en éclat." ${ }^{1}$ )

Und an anderer Stelle:

„... je démèle mal ici le subjectif et l'objectif et il me semble que le phénomène objectif et le phénomène subjectif sont en quelque sorte le même phénomène . . . “2)

Und endlich:

„Le phénomène objectif est probablement en relation étroite avec le phénomène subjectif." ${ }^{3}$ )

Bei der Zusammenfassung der Ergebnisse seiner zweiten Arbeit betont Bourdon, dass vor allem das subjektive Phänomen bei einer erheblicheren Verschiedenheit der Bilder die richtige Angabe ermöglicht, welches Auge das minderwertige Bild erhält bezw. umgekehrt. Unter gewissen, oben bereits angegebenen Umständen kann das objektive Phänomen die Unterscheidung unterstützen. Für das gewöhnliche binokulare stereoskopische Sehen (vision stéréoscopique birétinienne) kommen diese Phänomene nicht in Betracht; eine Unterscheidung der Eindrücke beider Augen ist also hier auch nicht möglich ${ }^{4}$ ).

Dieses sind in der Hauptsache die Versuche und Ergebnisse der Bourdon'schen Arbeiten. Auf einige weitere Einzelheiten einzugehen, wird sich sogleich noch Gelegenheit bieten.

Wir wollen nun im folgenden ein kurzes Resümee der Nachprüfung der Bourdon'schen Versuche geben, welches in vielen Punkten eine sehr erfreuliche Bestätigung der Ergebnisse bietet, freilich in mancher Hinsicht auch Abweichungen unserer Resultate erkennen lassen wird. Ausserdem glauben wir uns der Deutung, welche Bourd on einzelnen seiner Beobachtungen gegeben hat, nicht wohl anschliessen zu können.

Zunächst haben wir die Versuche im Dunkelzimmer wiederholt, bei welchen, während beide Augen den leuchtenden Punkt fixierten, derselbe für ein Auge verdeckt wurde.

Wir bedienten uns eines kleinen leuchtenden Punktes in einem Aubert'schen Diaphragma und verdeckten bald das eine, bald das

1) 1. c. p. 47 .

2) Ebenda p. 58.

3) Ebenda p. 55.

4) Hierin stimmen Bourd on und Heine überein. 
Nochm. zur Frage d. Unterseheidbarkeit rechts- u. linksäugiger Eindrücke. 271

andere Auge mit einem schwarzen Schirm. Da es sich für uns nur darum bandeln konnte, zu untersuchen, worauf etwa das „objektive Phänomen" Bourdon's beruhe, ob es sich wirklich um ein Lokalzeichen handelt oder nicht, haben wir diese Versuche wissentlich angestellt. Der Beobachter besorgte daher die Verdeckung selbst, indem er den Schirm vor das Auge schob. Zunächst wurde die Verdeckung nicht, wie Bourdon es stets bei seinen Versuchen getan hatte, von unten, sondern von der jeweiligen temporalen Seite des zu verdeckenden Auges her vorgenommen. In der Tat zeigte sich im Moment der Verdeckung eine partielle Verdunkelung des leuchtenden Punktes derart, dass von derjenigen Seite her, welche dem verdeckten Auge entsprach, sich ein Schatten über die eine Hälfte des den eigentlichen leuchtenden Punkt umgebenden Strahlenhofes (der sich im Dunkelzimmer nie ganz vermeiden lässt; s. u.) zu legen scheint. Dieser Schatten schnitt mit einer vertikalen Trennungslinie $a b$ und reichte dicht bis an das helle Zentrum heran.

Der erste Gedanke, der sich uns zur Erklärung dieser Erscheinung darbot, war, weil wir zunächst den leuchtenden Punkt zentral fixierten, dass die Erscheinung auf der, soviel uns bekannt, schon von Purkinje besehriebenen Beobachtung beruhe, der zufolge bei leuchtenden Punkten und Flächen im Dunkeln zuweilen vom Fixationspunkte nach dem blinden Flecke zu eine Art Krebsscherenfigur mit bogenförmigen Begrenzungslinien zu sehen ist ${ }^{1}$ ). Dadurch wäre ohne weiteres erklärt gewesen, warum der Schatten stets gleichseitig mit dem verdeckten Auge auftrat. Diese Erklärung musste aber fallen gelassen werden, da auch wir, wie bereits Bourdon, genau dasselbe Schattenphänomen im indirekten Sehen beobachten kounten ${ }^{2}$ ).

1) Diese Erscheinung ist besonders deutlich zu sehen, wenn man eine rote Leuchtquelle, z. B. ein glimmendes Streichhölzchen, im Dunkeln fixiert. Je nachdem man monokular oder binokular beobachtet, sieht man von der Leuchtquelle nach der einen oder nach beiden Seiten je zwei bläuliche Bogen zur Gegend des blinden Flecks ziehen.

2) Wir halten es nicht für ausgeschlossen, dass die "Krebsscherenfigur" bei dem einen Versuche Bourdon's (Bullet. etc. l. c. p. 10) das Urteil ermöglicht hat. Dabei benutzte Bourdon nämlich ein helles Quadrat von $3 \mathrm{~cm}$ Seite als Objekt, welches er im Dunkelzimmer fixierte. (Hier zeigen sich deutlich von den Ecken des Quadrats ausgehende bogenförmige Linien.) Bei der Verdeckung glaubte Bourdon nun wahrzumehmen, dass irgend etwas auf der Seite, welche dem verdeckten Auge entsprach, vor sich gehe: „Il me semble que la parti 
Wir glauben nun aber gleichwohl eine einfache Deutung geben zu können. Nahmen wir nämlich die Verdeckung des Auges nicht von aussen, sondern von innen, also von der Nasenseite her vor, so zeigte sich, dass nun der Schatten nicht auf der gleichseitigen, sondern auf der entgegengesetzten Seite auftauchte. Wurde die Verdeckung von unten her vorgenommen, so kam der Schatten ebendaher, wenn von oben, aus ebendieser Richtung ${ }^{1}$ ). Im letzten Falle bestand nur für das rechte Auge des einen von uns (Brückner) eine Abweichung insofern, als der Schatten öfters nicht, wie eigentlich zu erwarten gewesen wäre, von oben, sondern von unten her zu kommen sehien. Sehen wir zunächst von dieser letzten Tatsache $a b$, so ergibt sich die Erklärung eigentlich von selbst. Sie ist in der nicht momentanen, sondern sukzessiven Verdeckung der Pupille des einen Auges zu suchen. Infolgedessen werden diejenigen Strahlen, welche durch den zuerst verdeckten Teil der Pupille hindurehtreten, auch zuerst vom Einfall ins Auge abgehalten. 'Da ja infolge der relativen Unvollkommenheit des optischen Apparates unseres Auges eine absolut exakte Bilderzeugung auf der Retina unmöglich ist, so gibt es notwendigerweise von leuchtenden Punkten eine kleine Zerstreuungsfigur, welche dann als kleiner Stern mit zahlreichen, wenn auch sebr feinen und kurzen

gauche (bei Verdeckung des rechten Auges) du carré lumineux n'est pas éclipsé et qu'il se produit une ombre qui n'atteint que la partie droite du carré; en outre le phénomène d'obscurcissement paraît commencer à droite du carré lumineux et finir à gauche." Es mag sein, dass hier bei der Abgabe des Urteils der einseitige Fortfall der beiden Bogen (Bourdon stellte diesen Versuch nur bei direkter Betrachtung an) mitgewirkt hat. Auch wir konnten bei der Wiederholung dieses Versuchs zuweilen eine Art Schatten rechts oder links wahrnehmen, welcher von dem einseitigen Fortfall der hier deutlich wahrnehmbaren "Krebsscherenfigur" abhängig war. - Ausserdem aber müssen wir bemerken, dașs natürlich bei sọ grossen Objekten, wie es ein leuchtendes Quadrat von $3 \mathrm{~cm}$ Seite ist, ein langsames Verdecken eines Auges als allmählich fortschreitende Verdunkelung des Quadrates erkannt werden muss.

1) Noch deutlicher ausgesprochen ist die allmählich fortschreitende Verdunkelung, wenn man den leuchtenden Punkt durch Vorsetzen von z. B. + 15,0 D. vor das Auge in Form eines grossen Zerstreuungskreises auf der Netzhaut sich abbilden lässt oder einen solchen durch Vorhalten eines feinen Loches in einem schwarzen Schirm vor dem Auge erzeugt. In letzterem Falle muss dann natürlich eine grössere leuchtende Fläche beobachtet werden. (Auch ist hier die Richtung, in welcher der Schatten sich bewegt, nebenbei bemerkt, entgegengesetzt der Bewegungsrichtung des verdeckenden Schirmes, wenn dieser sich zwischen Auge und stenopäischer Lücke befindet.) 
Strahlen erscheint. Selbst wenn man die Punkte wenig hell macht, wie Bourdon eigens zu dem Zwecke tat, um nicht aus der Verschiedenheit der Zerstreuungskreise auf das eine oder andere Auge schliessen zu können, so lassen sich diese Strahlen doch nie ganz vermeiden.

Setzen, wir voraus, dass die Mehrzahl der in das Auge einfallenden Strahlen eines leuchtenden Punktes, sofern diese nicht genau vereinigt werden, einen gekreuzten Verlauf nimmt, so würde ein Strahlbündel, welches z. B. durch die temporale Pupillenhälfte geht, eine Spur nasal vom eigentlichen Bild auf die Netzhaut fallen. Es wäre dann ohne weiteres verständlich, dass, wenn dieses Strahlbündel durch einen Schirm am Eintritt in die Pupille gehindert wird, der entsprechende Strahl in der Zerstreuungsfigur fortfallen muss. Dieses wird an der durch binokulare Kombination entstandenen Sternfigur des Punktes als eine Art Schatten im Moment der Abblendung wahrzunehmen sein. Dass bei unseren Versuchen in einem Falle zuweilen der Schatten bei Verdeckung von oben her unten aufzutauchen schien, liesse sich ungezwungen erklären, wenn man für das Strahlbündel, welches den nach unten gehenden Hauptstrahl im Zerstreuungsbilde des einen Auges verursachte, einen ungekreuzten Verlauf annimmt ${ }^{1}$ ). Dass Bourdon bei seinen Versuchen, bei denen die Verdeckung stets von unten her erfolgte, den Schatten immer von aussen her auftauchen sah, mag vielleicht darauf beruhen, dass in seinen Augen die Zerstreuungsfiguren hauptsächlich in horizontaler Richtung derart ausgedehnt sind, dass für das rechte Auge das linke Ende der Figur höher steht als das rechte, während es sich im linken Auge umgekehrt verhält; in diesem Falle wäre gekreuzter Verlauf der Strahlenbündel im Auge anzunehmen. Es liesse sich aber auch denken, sofern die angenommene Form der Zerstreuungskreise unrichtig wáre, dass die jeweiligen äusseren, $d . \mathrm{h}$. temporal erscheinenden Strahlen durch die untere Pupillenhälfte ins Auge einträten. Auch wäre es möglich, dass im Bilde des rechten Auges die Strahlen vorzugsweise auf der rechten, im linken Auge auf der linken Seite des zentralen Kerns des Punktes vorhanden waren.

Bei diesen Versuchen ist ferner zu berücksichtigen, dass die Pupillen im Dunkelzimmer ja sehr stark erweitert sind, wodureh die sphärische Aberration und damit die Zerstreuungsfiguren um den

1) Worin die Ursache für denselben im konkreten Falle zu suchen ist, müssen wir unentschieden lassen.

E. Pflüger, Archiv für Physiologie. Bd. 107. 
leuchtenden Punkt zunehmen müssen. In der Tat zeigte sich, dass bei eserinisiertem Auge, also bei maximal verengter Pupille das Bild einer kleinen Öffnung im Aubert'schen Diaphragma ungleich schärfer erschien. Es zeigten sich z. B. für das rechte Auge des einen von uns (Brückner) nur nach rechts hin zwei deutliche Strahlen, während sonst die Grenzen nahezu scharf erschienen. Wurde nun - bei geschlossenem linkem Auge - die Verdeckung von den verschiedenen Seiten langsam vorgenommen, so war ein gleichsinniges Auftauchen der Verdunkelung deutlich erkennbar. Analoges, nur schwerer, war zu beobachten, wenn das linke Auge ebenfalls geöffnet war, - vorausgesetzt, dass die Verdeckung des rechten Auges lang. sam erfolgte. Wurde dieselbe dagegen schnell vorgenommen, so erschien stets, gleichgültig, von welcher Seite her dieselbe erfolgte, ein Schatten auf der rechten Seite der leuchtenden kleinen Öffnung, offenbar bewirkt durch den Fortfall der beiden im rechten Auge (wegen der Enge der Pupille) allein deutlich sichtbaren nach rechts von dem Leuchtpunkt sich ausdehnenden Strahlen.

Entsprechend der von uns im vorstehenden gegebenen Erklärung beobachteten wir bei Fixation des unteren von zwei übereinanderstehenden Punkten und Verdeckung des oberen Punktes für ein Auge, dass der Schatten aus der Richtung aufzutauchen schien, aus welcher die Verdeckung erfolgte. Es findet sich darin eine Abweichung von der Beobachtung Bourdon's, welcher in diesem Falle fand, dass der Schatten von der entgegengesetzten Seite zu kommen schien. Bourdon glaubte seine Beobachtung durch die geringe Lageveränderung erklären zu können, welche die scheinbare Vertikale bekanntermassen erfährt, je nachdem mit dem rechten oder linken oder mit beiden Augen gesehen wird. Es mag sein, dass die nicht unerheblich schwierigere Beobachtung im indirekten Sehen, welche sich in gleicher Weise auch bei zwei horizontal nebeneinander befindlichen Punkten, von denen der indirekt gesehene für ein Auge verdeckt wird, bemerklich macht, bei der mangelnden Übereinstimmung der Resultate mitgewirkt hat. Ausserdem mögen ja die feineren Refraktionsverhältnisse für diejenigen Strahlen, welche indirekt auf die Netzhaut fallen, von den für die zentralen gültigen individuell sehr verschiedenartige Abweichungen zeigen ${ }^{\text {}) . ~}$

1) Man vergleiche zu den vorstehenden Ausführungen insbesondere Helm holtz, Physiol. Optik. 2. Aufl. S. $170 \mathrm{ff}$. 
Für diejenigen Versuche Bourdon's im Dunkelzimmer, bei welchen gleich zu Beginn der Beobachtung das eine Auge ganz oder teilweise vom Sehakte ausgeschlossen war, findet sich eine vollkommene Bestätigung bereits in unseren unabhängig von Bourdon angestellten Experimenten, welche früher bereits mitgeteilt wurden, und wir benutzen sehr gerne die Gelegenheit, die Priorität Bour don's in der Beobachtung der auch von uns gefundenen Sensation festzustellen. Es erscheint uns aber für die Beurteilung der hier besprochenen Frage nicht unwichtig, nachdrücklichst zu betonen, dass von zwei verschiedenen Seiten unabhängig voneinander diese Sensation, welche von Bourdon als "subjektives Phänomen" bezeichnet worden ist, (neben anderen Momenten) als massgebend für die richtige Entscheidung, mit welchem Auge gesehen wird, erkannt worden ist. Wenn demgegenüber Heine trotz der Bekanntschaft mit der ersten Bourdon'schen Arbeit in seiner jüngsten Veröffentlichung die Meinung ausspricht, dass ihm bei seinen Versuchen diese Empfindung nicht so auffallend gewesen sei, dass er "die Unterscheidbarkeit rechtsund linksäugiger Eindrücke bei Ausschluss eines Auges vom Sehakt dadurch erklären zu können glaubte" ${ }^{1}$ ), so muss es sich wohl hierbei um individuelle Differenzen in der Empfindlichkeit für das Phänomen handeln. Wir haben darauf auch bereits in unserer zweiten Arbeit hingewiesen ${ }^{2}$ ).

Auch in der Beziehung mögen wohl individuelle Verschiedenheiten vorkommen, dass dem einen Beobachter sich das am verdeckten bezw. weniger sehenden Auge auftretende Gefühl der Schwere usw. besonders bemerklich macht, wie es bei uns der Fall ist, während die von Bourdon ausserdem beobachtete Empfindung der Leichtigkeit im allein bezw. mehr sehenden Auge demgegenüber von untergeordneter Bedeutung erscheint. In der Tat können wir auch jetzt die letztere Sensation wirklich deutlich nicht wahrnehmen.

1) Pflüger's Arch. Bd. 101 S. 68.

2) Pflüger's Arch. Bd. $91 \mathrm{~S} .362 \mathrm{f}$. Dort ist auch gesagt, dass sich in dieser Hinsicht Unterschiede zwischen den beiden Augen einer und derselben Versuchsperson ergeben haben - eine Tatsache, welche auch von Bourdon bei einem seiner Versuche festgestellt wurde. Er sagt (Bullet. etc. l. c. p. 17): „Je distinguais mieux la sensation de lourdeur de l'œil droit quand il ne voyait pas le point que celle de l'œil gauche." 
Bourdon gibt ja ebenfalls an, dass er bei seinen ersten Versuchen zuerst bemerkt habe, mit welchem Auge er nicht sieht ${ }^{1}$ ).

Wenn sich somit hinsichtlich der beobachteten Tatsachen durchaus Übereinstimmung zwisehen B ourd on und uns ergibt, so können wir uns aus sogleich näher darzulegenden Gründen der Deutung, welche Bourdon diesem Phänomen gibt, zu unserem Bedauern nicht anschliessen. Wir hatten in unseren Untersuchungen auf Grund der Tatsache, dass sich peripher kein Anhaltspunkt für die Entstehung desselben ermitteln liess, dasselbe als zentral bedingt angesprochen und aus gleichem Grunde die Bezeichnung "scheinbares" Organgefühl angewendet. Bourdon hat nun unserer Auffassung gegenüber auch in seiner letzten Arbeit an seiner bereits früher ausgesprochenen Ansicht, dass es sich um ein Muskel- und Sehnengefühl handle, festgehalten. Er sagt darüber folgendes:

"A mon avis, il s'agit ici d'une sensation fournie par les muscles des yeux (ou leurs tendons). Pour moi, en effet, la sensation qui se produit dans l'oil où l'impression a la moindre valeur peut être assez exactement décrite comme une sensation de lourdeur, et celle qui se produit dans l'œil où l'impression a la plus grande valeur comme une sensation de légèreté, c'est-à-dire que ces sensations ressemblent' à celles qu'on éprouve, par exemple, en soulevant un poids lourd ou léger; or celles-ci! (abstraction faite des sensations de pression qui peuvent les accompagner) sont vraisemblablement des sensations des muscles ou des tendons. On peut supposer que la sensation de lourdeur, par exemple, qui se produit dans l'œil qui reçoit l'image la plus sombre ou la moins nette, tient à ce que l'appareil musculaire de l'œil considéré se trouve, comparé à celui de l'autre oeil, dans un état de dépression relative qui fait que l'effort de fixation est senti plus fortement par cet oil que par l'autre. Cet état de dépression a lui même pour cause la faiblesse de l'excitation rétinienne. Que l'état de contraction des muscles des yeux soit sous la dépendance de l'excitation rétinienne, c'est ce qu'il me paraît inutile de s'attarder à démontrer ${ }^{2) . " ~}$

Gegenüber der vorstehenden Ansicht B ourdon's möchten wir doch einige Bedenken geltendmachen, welche sich zum Teil auf bekannte Anschauungen, zum Teil auf von uns beobachtete Tatsachen gründen. In erster Linie sei hier die wohl allgemein verbreitete Ansicht von der gleichsinnigen und gleichstarken Innervation

1) Auch Hein e meint, dass dem einen die Reizung des einen Auges, dem andern aber die Kontrastwirkung, als welche er unser Abblendungsgefühl aufzufassen geneigt ist, als das Wesentliche erscheinen könne. (Pflüger's Arch. Bd. 101 S. 68.)

2) L'année psychologique 1903 p. 54. 
der Muskeln beider Augen erwähnt ${ }^{1}$ ), welche sich unter anderem auf die Tatsache der primären und sekundären Schielablenkung bei paralytischem Schielen stützt. Nach den bisherigen Erfahrungen kann auch der okulomotorische Apparat in genau gleicher Weise von nur einer wie von beiden Netzhäuten aus in Tätigkeit versetzt werden.

Wenn Bourdon bei seiner Erklärung offenbar davon ausgeht, dass er in dem Auge, welches das minderwertige Bild empfängt, ein Gefühl von Schwere, im anderen dagegen ein Gefühl von Leichtigkeit empfindet, so steht dem gegenüber die Beobachtung einzelner unserer Versuchspersonen, welche ausschliesslich die Empfindung zu haben angaben, als ob das Oberlid des einen Auges herabgesunken wäre, als ob das Auge leicht geschlossen sei $^{2}$ ).

Aber gerade in dieser verschiedenartigen Beschreibung und Auffassung, welche die doch offenbar bei allen Beobachtern mehr oder weniger identische Sensation erfährt, glauben wir einen Grund dafür sehen zu müssen, dieselbe als zentral bedingt und nicht als peripher in den Muskeln und Sehnen entstehend aufzufassen. Auch möchten wir an die von uns früher beschriebene Beobachtung erinnern, der zufolge sich bei Vorhandensein eines monokularen positiven Nachbildes im Dunkelraume bei geschlossenen Augen die Empfindung einstellte, als ob nur das eine Auge wirklich geschlossen sei, das Nachbildauge dagegen geöffnet wäre ${ }^{3}$ ).

Sodann sei auch der Umstand erwähnt, dass schon eine geringe Differenz in der Helligkeit der Bilder beider Augen genügen kann, um das "Abblendungsgefühl" auf dem einen Auge hervorzurufen. Dabei aber braucht die Helligkeit des einen Bildes nur um so wenig geringer zu sein, dass weder eine merkliche Herabsetzung der Sehschärfe noch irgendwelche Erschwerung in der Fixation einzelner Objekte, noch auch irgendwelche hemmenden Momente für eine binokulare Verschmelzung sich bemerkbar machen.

Wenn wir demnach mit Rücksicht auf die angeführten Gründe nicht in der Lage sind, unsere früher ausgesprochene Ansicht von

1) Man vergleiche hierzu: Hering, Zur Lehre vom binokularen Sehen S. 2 ff. Leipzig 1868.

2) Wir wollen allerdings nicht verfehlen, darauf hinzuweisen, dass andere unserer Beobachter auch die Empfindung hatten, als ob das eine Auge nicht so frei bewegt werden könnte wie das andere.

3) Pflüger's Arch. Bd. 91 S. $368 \mathrm{f}$. 
der zentralen Bedingtheit des Gefühles zugunsten der Bourdonschen Auffassung aufzugeben, so möchten wir doch nochmals betonen, dass es sich hier ja nur um die verschiedene theoretische Auffassung einer im grossen und ganzen von beiden Seiten vollkommen übereinstimmend und unabhängig voneinander beobachteten $\mathrm{Tat}$ sache handelt, und dass beide Teile zu der Ansicht gekommen sind, es handle sich unter bestimmten, näher angegebenen Bedingungen, bei welchen eine Unterscheidung des rechten und linken Auges möglich ist, nicht um ein spezifisches Lokalzeichen, sondern um eine Unterscheidung auf indirektem Wege.

Nur nebenbei möchten wir bemerken, dass wir nach der von uns gegebenen Erklärung des objektiven Phänomens Bourdon's nicht in der Lage sind, uns der oben zitierten Anschauung, dass ein enger Zusammenhang zwischen dem "objektiven" und dem "subjektiven" Phänomen (im Sinne Bourdon's) bestehe, anzuschliessen.

Mit kurzen Worten sei noch der Versuche mit dem Stereoskop gedacht, welche wir in ähnlicher Weise anstellten, wie Bourdon es getan hatte (cf. oben S. 269 f.). Waren die Öffnungen, welche binokular verschmolzen wurden, sehr klein, so war für uns selbst, sofern der durch die eine Öffnung sichtbare helle Grund durch eine dunklere Farbe ersetzt wurde, zuweilen eine Andeutung des „Abblendungsgefühls" wahrzunehmen, während wir von einem Schatten, der sich vor das eine Auge zu legen scheint, nichts bemerken konnten, wie Bourdon und auch eine unserer Versuchspersonen ihn wahrzunehmen imstande waren.

Verwendeten wir dagegen etwas grössere Öffnungen $(7 \mathrm{~mm})$, als Bourdon es getan hatte $(0,5$ und $2 \mathrm{~mm})$, so machte unsere eben erwähnte Versuchsperson lauter unrichtige Angaben; der Schatten erschien ihr stets auf der falschen Seite. Bei ihr stellte sich nämlich unter diesen Bedingungen (genau wie auch bei uns) im Momente der Verdeckung der einen Öffnung durch die andere Farbe ein partieller Zerfall in Doppelbilder ein, der offenbar durch einen Fortfall des Fusionszwanges bedingt war. Wie sich bei genauer Prüfung herausstellte, waren die Doppelbilder gekreuzt, was seine Erklärung in einer ungenügenden Konvergenz der Gesichtslinien bei der Anstellung des Versuches findet. Wenn es uns bei unseren eigenen Beobachtungen gelang, einen Zerfall in Doppelbilder zu verhindern, so waren wir freilich auf Grund des Abblendungsgefühles, welches hier deutlicher auftrat als bei den kleineren Öffnungen, meist imstande, richtig an- 
zugeben, welches Auge verdeckt wurde. Von irgendeinem Schattenphänomen, analog demjenigen im Dunkelzimmer, konnten wir aber anch bei den grösseren öffnungen nichts wahrnehmen. Wir sind daher geneigt, anzunehmen, dass es sich vielleicht bei dem hier von Bourdon beobachteten Schattenphänomen um etwas anderes handelt als bei demjenigen im Dunkelzimmer. Wir halten es nämlich nicht für ausgeschlossen, dass auch bei Bourdon ein partieller Zerfall in gleichnamige Doppelbilder stattfand. War nun der Grund z. B. weiss, die verdeckende Farbe blau, so behielt das Weiss in demjenigen Teil des Sehfeldes, in welchem die Doppelbilder sich noch deckten, im Wettstreit das Übergewicht. Der übrige Teil des einen Doppelbildes, welches jetzt blaue Färbung zeigte, musste dann als relatives Dunkel auf der betreffenden Seite erscheinen ${ }^{1}$ ).

Wir hatten früher ${ }^{2}$ ) des näheren auseinandergesetzt, dass als Ursache, durch welche das Abblendungsgefühl in dem einen Auge ausgelöst werden kann, sowohl die geringere Helligkeit des einen Bildes als auch seine geringere Schärfe nachzuweisen ist. Im folgenden möchten wir noch über einen Versuch berichten, in welchem bald das eine bald das andere Moment die Entstehung der Sensation verursachte. Es wurde z. B. das rechte Auge bei mässig hellem Wetter homatropinisiert ${ }^{3}$ ). Nachdem die Pupille weit geworden, wird ein starkes Abblendungsgefühl auf dem rechten Auge wahrgenommen. Bei abwechselndem Schliessen der Augen lässt sich feststellen, dass das Bild des rechten Auges viel undeutlicher ist. Beim Lesen in der Nähe obne Korrektion (Refraktion: rechts $-3 \mathrm{D}$,

1) Das Phänomen des binokularen Wettstreites, welches Bourdon sowohI hier wie bei anderen seiner Versuche zu beobachten Gelegenheit hatte, erklärt wohl auch ungezwungen die von Bourdon (L'année psychologique 1903 p. 45) beobachtete Tatsache, dass im Dunkelraume eine feine Leuchtlinie, welche zunächst binokular sichtbar ist, nach dem Unsichtbarwerden für das eine Auge öfters im ersten Moment gänzlich verschwindet. Wahrscheinlich gewinnt hier, unterstützt durch die grössere oder geringere lokale Adaptation in dem allein weitersehenden Auge, das succesive Kontrastschwarz in dem anderen Auge momentan das Übergewicht. Aus diesem Grunde ist es auch verständlich, dass die besprochene Erscheinung (auch bei leuchtenden Punkten) peripher deutlicher auftritt wie zentral, wegen der dort stärker ausgesprochenen Adaptationsfähigkeit (Ermüdbarkeit).

2) Pflüger's Arch. Bd. 91 l. c.

3) Als Beispiel wählen wir einen einzelnen Versuch. 
links - 2,25 D.) wird das Abblendungsgefühl auf dem rechten Auge wesentlich schwächer, um sich bei Annäherung des Buches über den Fernpunkt heran rechts wieder zu verstärken: das Bild des rechten Auges erscheint wegen der aufgehobenen Akkommodation viel undeutlicher. Wird das korrigierende Glas vorgesetzt, so verschwindet beim Umhersehen im Zimmer das Abblendungsgefühl auf dem rechten Auge sofort, ist dagegen links schwach vorhanden: das Bild des rechten Auges erscheint bedeutend heller (wegen der weiteren Pupille). Beim Lesen in der Nähe mit Korrektion Abblendungsgefühl rechts: das Bild des rechten Auges ist wegen der Unmöglichkeit zu akkommodieren ganz verwaschen. - Wurde kurze Zeit der Blick gegen den mässig hellen Himmel gerichtet, so erschien nach dem Eintritt in ein halbdunkles Zimmer das Abblendungsgefühl rechts: das Bild des rechten, infolge der weiten Pupille stärker hell adaptierten Auges erscheint viel dunkler. Nach beiderseitiger Dunkeladaptation macht sich beim Hinaustreten ins helle Zimmer links das Abblendungsgefühl bemerkbar: das Bild des linken Auges ist - infolge der engeren Pupille - dunkler.

Darauf wurde in das rechte homatropinisierte Auge ein Tropfen Eserin gegeben, dessen Wirkung sich nach einigen Minuten durch Zucken im M. orbicularis zu erkennen gab. Nach Ablauf von zehn Minuten war mit Korrektion beim Lesen in der Nähe rechts nur noch eine Spur des Gefühls zu konstatieren: infolge Wiederkehr der Akkommodation sind die Bilder beider Augen gleich deutlich. Ohne Korrektion ist das Gefühl rechts noch schwach vorhanden. Im hellen und halbdunklen Zimmer hat sich in der Lokalisation des Gefühls mit und ohne Korrektion - noch nichts gegen früher geändert: die rechte Pupille ist noch fast maximal weit.

Nach Verlauf von ca. 20 Minuten nach der Einträufelung des Eserins war die maximale Wirkung desselben auf den Ciliarmuskel eingetreten. Bereits durch eine geringe Akkommodationsinnervation gelang es, maximale Ciliarmuskelkontraktion zu erreichen. Wurde nun mit dem korrigierenden Glase ein entferntes Objekt im hellen Zimmer fixiert, so war zunächst links eine schwache Empfindung vorhanden, welche aber sofort versehwand und dafür rechts erschien, wenn ohne Änderung der Blickrichtung willkürlich eine leichte Akkommodationsinnervation intendiert wurde ${ }^{1}$ ). Durch letztere

1) Bei einiger Übung ist das bekanntlich auch ohne Fixierung eines nahen Objektes möglich. 
wurde rechts nämlich ein viel stärkerer Effekt erzielt als links, infolgedessen war auch das Bild des rechten Auges viel verwaschener als das des linken. Wurde nun der Akkommodationsimpuls wieder unterbrochen, so nahm das Gefühl auf dem rechten Auge in genauer Parallele mit dem durch das Eserin verlangsamten Nachlassen der Akkommodation und damit einhergehender, zunehmender Deutlichkeit des Bildes des rechten Auges ab, um schliesslich ganz zu verschwinden und schwach wieder links aufzutreten, - das Bild des linken Auges war ja dunkler.

Die vorstehend mitgeteilte Versuchsreihe wurde dann abgebrochen, weil wegen zu geringer Menge des Eserins maximale Pupillenverengerung nicht eintrat. War dieselbe dagegen eingetreten, wie es bei analogen Versuchen der Fall war, so verursachte nun die geringere Weite der Pupille und die dadurch bedingte geringere Helligkeit des Bildes ein deutliches Abblendungsgefühl auf dem betreffenden Auge, gleichgültig, ob die korrigierenden Gläser aufgesetzt wurden oder nicht.

Bei dem beschriebenen Versuche handelt es sich offenbar um ein Interferieren zweier Momente, welche das Abblendungsgefühl auslösen können: nämlich der scheinbaren Helligkeit und der ungleichen Schärfe der Bilder beider Augen.

Als letzter Punkt unserer Ausführungen bleibt uns noch die Auseinandersetzung mit der Entgegnung Heine's auf unsere beiden früheren Mitteilungen.

Heine war in seiner ersten Arbeit ${ }^{1}$ ) zu dem Ergebnis gekommen, dass sich die Wabrnehmungen der linken Fovea von denen der rechten unterscheiden lassen, und zwar hatte er angenommen, dass dem sinnlichen Eindruck als solchem die Eigenschaft der Unterscheidbarkeit anhaftet, während wir eine solche nicht zugeben konnten ${ }^{2}$, da es uns bei allen unseren Versuchen, sofern ein richtiges Urteil in der angegebenen Richtung möglich war, gelang, indirekte Momente als massgebend für das Urteil festzustellen. Wir hatten ferner mitgeteilt, in welcher Weise es uns möglich war, ein richtiges Urteil darüber, welches Auge allein bei Ausschluss des anderen sieht, zu verhindern.

1) Klin. Monatsbl. f. Augenheilk. Jahrg. 39 Heft 2 S. 615 ff.

2) Pflüg er's Arch. Bd. 90 S. 290 ff. 


\section{Heine sagt nun am Eingang seines letzten Aufsatzes ${ }^{1}$ ):}

„Das Wesentiche der damaligen Mitteitung (scil. Heine's) war der Nachweis, dass bei Ausschluss eines Auges vom Sehen und bei Reizung des anderen Auges durch ein Lichtpünktchen mir eine Entscheidung möglich ist, welches Auge das sehende ist. Wie wir sehen werden, haben Br ückner und v. Brücke dieses Resultat bestätigt, nur ihre Deutung ist eine andere. In dieser Hinsicht hatte ich mir nur diese Bemerkung erlaubt: ,Soweit man der Selbstbeobachtung in solchen Fragen, welche der experimentellen Erforschung Schwierigkeiten entgegensetzen, trauen kann, möchte ich annehmen, dass dem sinnlichen (centripetalen) Eindruck als solchem die Eigenschaft der Unterscheidbarkeit anhaftet." Die beiden Herren Verfasser bestreiten dieses und nehmen statt dessen ein Organgefüht im nicht sehenden Auge an."

Dem gegenüber sei bemerkt, dass aus der ersten Arbeit Heine's insofern doch nicht klar hervorging, dass das Wesentliche derselben die Unterscheidbarkeit fovealer Eindrücke bei völligem Ausschlusse eines Auges vom Sehakte war, als unter 7 und 8 auch Versuche angeführt wurden, in welchen beide Augen am Sebakte beteiligt waren. Heine sagt denn anch im Anschluss an die Mitteilung desselben:

"Namentlich aus diesen letaten Versuchen geht hervor, dass die Entscheidung, welches Auge das sehende ist, um so schwerer ist, je ähnlicher der rechtsäugige Eindruck dem linksäugigen ist, am leichtesten ist es, wenn das eine Auge gar nichts sieht.

Nach den geschilderten Versuchen, glaube ich annehmen zu sollen, dass sich die Wahrnehmungen der linken Fovea von denen der reehten sehr wohl unterscheiden lassen, im speziellen ${ }^{2}$ ), dass man sehr wohl in der Lage ist, zu sagen, welches Auge das sehende ist, wenn nur einem Auge ein Leuchtpunkt geboten wird, das andere dabei völlig unbelichtet ist $\left.{ }^{3}\right) . "$

Ausserdem verdichtet sich die oben zitierte nur hypothetisch und mit grosser Reserve ausgesprochene Deutung, welche $\mathrm{He}$ ine für die Unterscheidbarkeit angibt, am Sehlusse seines ersten Aufsatzes zu folgendem sehr viel bestimmter gehaltenen Satze:

"Wenn wir also nach den obigen Versuchen die Existenz einer retinalen Unterscheidbarkeit rechts- und linksäugiger Wahrnehmungen anerkennen müssen, so ist dieser eine Bedeutung für das körperliche Sehen doch keineswegs zuzusprechen $\left.{ }^{4}\right) . "$

1) Pflüger's Arch. Bd. 101 S. 67.

2) Im Original nicht gesperrt gedruckt.

3) Klin. Monatsbl. f. Augenheilk. 1. c. 619.

4) 1. c. S. 620 . 
Nochm. zur Frage d. Unterscheidbarkeit rechts- u. linksäugiger Eindrücke. 283

Es kann darum wohl nicht als ungerechtfertigt erscheinen, wenn wir bei unseren Untersuchungen dieser theoretischen Auffassung Heine's gegenüber betonten, dass eine Unterscheidung im angegebenen Sinne, sofern sie überhaupt zustande kommt, stets nur durch indirekte Momente ermöglicht wird ${ }^{1}$ ). Wenn Heine als solches von uns angegebenes Moment nur das Organgefühl anführt, so müssen wir feststellen, dass wir ausser demselben noch zwei andere Ursachen angeführt haben, welche $\mathrm{zunächst} \mathrm{die} \mathrm{Unter-}$ scheidung gestatten können (s. u.). -

Vor allem aber haben wir uns gegen die Behauptung Heine's zu verwahren, als hätten wir einen durch unsere Experimente nicht bewiesenen Satz behauptet. Heine sagt ${ }^{2}$ ):

"Wenn am Schlusse der Arbeit (scil. unserer) gesagt wird: „. . . so ist es auch bei völligem Ausschluss des einen. Auges vom Sehalite unmöglich anzugeben, ob eine Gesichtswahmehmung durch das rechte oder linke Auge vermittelt werde," so ist dieser Satz durch die Experimente nicht bewiesen. Eine Reihe von Versuchen, welche diese Behauptung beweisen sollen, wurden in der Weise angestellt, dass auch dem angeblich nicht sehenden Auge grössere Leuchtflüchen gezeigt wurden, wodurch der Beobachter dann natürlich getäuscht wurde."

Zunächst lautet unser von Heine nur halb zitierter Satz im ganzen folgendermassen:

„Wenn es gelingt, diese Faktoren auszuschliessen, wie wir es bei unseren Versuchen gethan haben, so ist es auch bei völligem Ausschluss etc. $\left.{ }^{3}\right) . "$

Welche Faktoren auszuschliessen sind und wie das geschieht, haben wir in unserer ersten Arbeit im Verlaufe der Darstellung unserer Versuche ausgefuhrt. Wir haben zunächst, da eine Reihe

1) Wenn Heine in seiner letzten Veröffentlichung sagt: „Schliesslich sagen die Verfasser: ,Somit bestätigt sich also die Angabe von Helmholtz, dass wir für gewöhnlich kein bestimmtes Beurusstsein davon haben, mit welchem Auge wir das eine oder andere Bild sehen." Dies habe ioh nirgends bestritten" (Pflüger's Arch. Bd. 101 S. 69), so möchten wir dem gegenüberhalten, dass wir den Nachdruck nicht auf diese erste, allein von $\mathrm{He}$ ine zitierte Hälfte des Satzes von Helmholtz legten und legen, sondern auf die zweite, mit folgenden Worten unmittelbar anschliessende: $\ldots$. und dass wir dies ,nicht oder nur unvollkommen und nur durch nebensächliche Umstände zu beurtheilen" wissen". (Pflüger's Arch. Bd. 90 S. 302). Der Gegensatz liegt also anch hier wieder darin, dass $\mathrm{H}$ eine eine retinale Unterscheidbarkeit annimmt, dagegen von $\mathrm{Helm}$ holtz, Bourdon und uns indirekte Momente aufgezeigt worden sind.

2) Pflüger's Arch. Bd. 101 S. 68.

3) Pfl üger's Arch. Bd. 90 S. 302. 
von Versuchspersonen das richtige Urteil auf Grund der relativen Rechts- oder Linkslage des leuchtenden Punktes abzugeben imstande war, durch Vorsetzen von horizontal ablenkenden Prismen eine Fälschung des Urteils bei manchen Beobachtern erzielen können. Das Protokoll einer besonders charakteristischen Versuchsreihe teilten wir mit ${ }^{1}$ ). Ausserdem wurde die einseitige Erhellung des Gesichtsfeldes und schliesslich das Organgefühl als bestimmend für das richtige Urteil erkannt. Wir haben nun ausdrüeklich angegeben, in welcher Weise es möglich war, auch diese Faktoren auszuschliessen. Pflüger's Archiv Bd. 90 S. 297 f. sagen wir:

„Befand sich die Versuchsperson längere Zeit im Dunkelzimmer, waren also beide Augen relativ gut dunkeladaptiert, wie es bei den ersten Versuchsreihen meist der Fall war, so musste offenbar schon eine sehr schwache einseitige Belichtung, wie sie der Leuchtpunkt im A u bert'schen Diaphragma bewirkte, die Entstehung des Gefühls hervorrufen können. Deshalb verliess bei weiteren Versuchen die Versuchsperson nach jedem Einzelversuch das Dunkelzimmer und blickte nach dem Himmel. Darauf wurde der nächste Versuch möglichst schnell gemacht und das Urtheil sofort nach dem Öffnen der Augen bei möglichster Verkleinerung des Punktes, welche für das nun hell adaptierte Ange natürlich nicht so weit gehen durfte wie sonst, abgegeben. Es zeigte sich, dass bei gleichzeitiger Anwendung von Prismen, um die Erkennung aus der scheinbaren Richtung auszuschliessen, nun das Urtheil in den meisten Fällen entweder unmöglich oder falsch wurde. Nach etwas längerem Aufenthalte im Dunkelzimmer und fortgesetzter Betrachtung des leuchtenden Punktes entwickelte sich dann das Abblendungsgefühl, und das Urtheil wurde richtig abgegeben.

In derselben Weise liess sich auch der Einfluss der auf der Seite des offenen Auges sich weiter erstreckenden diffusen Erhellung des Gesichtsfeldes ausschliessen, denn wie wir sahen, wurde auch diese un so deutlicher wahrgenommen, je stärker dunkel adaptiert das Auge war."

Es handelt sich also dem Vorstehenden zufolge um ganz andere Versuche, als um diejenigen, von denen Heine in seinem oben zitierten Satze behauptet, wir hätten sie zum Beweise der Unmöglichkeit der Unterscheidung angestellt. Jene Versuche mit den indirekt gesehenen Leuchtflächen dienten einem ganz anderen Zwecke. Wie nämlich ausdrücklich am Anfang des betreffenden Abschnittes gesagt ist, hatten wir sie angestellt, „um zu entscheiden, ob bei den Versuchen im Dunkelzimmer die infolge der Diffusion des Lichtes auftretende schwache Erregung des monocularen Gesichtsfeldtheils das Abblendungsgefühl auslöse . . . 2)." -

1) PfI ùger's Arch. Bd. 90 S. $294 \mathrm{f}$.

2) Pflü ger's Arch. Bd. 90 S. 299. 
Nochm. zur Frage d. Unterscheidbarkeit rechts- u. linksäugiger Eindrücke. 285

Nachdem wir Versuche mitgeteilt hatten, in welchen eine Fälschung des Urteils durch Vorsetzen von Prismen möglich war, sagen wir folgendes:

„Auf Grund dieser und ähnlicher Versuche glauben wir es nur einem ungünstigen Zufall zuschreiben zu müssen, dass Heine eine Fälschung des Urtheils durçh Prismen nicht erreichen konnte.

Mehrere Versuchspersonen waren aber trotz der Anwendung von Prismen in der Lage, fast ausnahmslos richtig zu erkennen, welches Auge bei Ausschluss des anderen vom Sehakte das sehende war ${ }^{1}$."

Es ist wohl zunächst nicht recht ersichtlich, inwiefern $\mathrm{Heine}$ an diesen beiden zitierten Sätzen Anstoss nehmen konnte, und zwar, wie wir zu verstehen glauben, aus dem Grunde, als hätten wir uns in denselben eines Widerspruches schuldig gemacht. Die Erklärung liegt vielleicht in folgendem: Aus der ersten Arbeit Heine's war nicht mit Bestimmtheit zu ersehen, ob er die mitgeteilten Versuche nur an sich oder auch an anderen Versuchspersonen angestellt habe. Da bei derartigen subtilen subjektiven Beobachtungen allgemeingültigere Ergebnisse natürlich nur zu erzielen sind, wenn man sich nicht ausschliesslich auf die Selbstbeobachtung beschränkt, so nabmen wir an, dass Heine an einer grösseren Zahl von Versuchspersonen experimentiert habe. In dieser Annahme aber haben wir uns offenbar getäuscht, denn Heine sagt selbst in seiner letzten Veröffentlichung folgendes:

„Das Wesentliche der damaligen Mitteilung war der Nachweis, dass bei Ausschluss eines Auges vom Sehen und bei Reizung des anderen Auges durch ein Lichtpünktchen mir eine Entscheidung möglich ist, welches Auge das sehende ist ${ }^{2}$ )."

und an anderer Stelle:

"Ich glaube, durch meine mitgeteitten acht Versuchsreihen gerade das Gebiet der Bedingungen scharf genug abgegrenzt $z u$ haben, unter denen ir $^{3}$ ) eine Unterscheidung, welches Auge das sehende sei, möglich ist $\left.{ }^{4}\right) . "-$

Wir haben bei unseren früheren Versuchen gefunden, dass die diffuse mehr nach der einen Seite gehende Erhellung des Gesichtsfeldes in vielen Fällen das richtige Urteil ermöglichte. Heine glaubte nun diesen Faktor durch Vorsetzen von Röhren ausschliessen zu
1) Pflüger's Arch. Bd. 90 S. 296.
2) Pflüger's Arch. Bd. 101 S. 67.
3) Dieses "mir" bei Heine gesperrt gedruckt.
4) Ebenda S. 70. 
können. Er ging dabei offenbar von der Voraussetzung aus, dass diese diffuse Erhellung durch Reflexion des Lichtes an den Staubteilchen der Zimmerlnft entstehe, während wir ausdrücklich dieselbe auf die Zerstreuung des Lichtes im Auge zurückgeführt haben. Wir schrieben ${ }^{\mathbf{1}}$ ):

„Da aber unter diesen Bedingungen anch bei sehr verkleinerter öffnung immer verhältnissmässig viel diffuses, infolge der nicht absoluten Klarheit der brechenden Medien im Auge zerstrentes Licht sich im Gesichtsfelde verbreitete, ..." und $\left.^{2}\right)$ :

„Diese Belichtung (des nasalen Netzhautteiles) ist eine Folge der Zerstreuung des Lichtes im Auge, weil seine brechenden Medien nicht absolut klar sind."

Eine Wiederholung des Versuches mit Röhren in der von Heine angegebenen Weise hat uns von neuem von der Richtigkeit unserer früheren Ansicht überzeugt. Im übrigen erlauben wir uns noch besonders zu betonen, dass es auf die diffuse mehr nach der einen Seite ausgedehnte Erhellung des Gesichtsfeldes ankommt, auf welchen Punkt $\mathrm{Heine}$ in seiner Erwiderung gar nicht näher eingeht. Es ist also nicht die Belichtung des peripheren Gesichtsfeldes als solche, sondern die entsprechende Lokalisation des Lichtscheines im Aussenraume massgebend.

Deshalb passt auch der von Heine (Pflüger's Archiv Bd. 101 S. 69) angegebene Versuch nicht recht hierher. Er sagt:

"Man kann aber auch isolirt die Wirkung der diffusen Belichtung eines Auges untersuchen ohne fovealen Eindruck, indem man nach einer roten Glühlampe blickt, wobei das eine Auge durch ein rotes, das andere durch ein grünes Glas sieht. Passen die Gläser gut zueinander, so wird der Lichteindruck von dem einem Auge fast völlig ferngehalten, das andere Auge bekommt einen starken Lichtreiz, welcher auch bei geschlossenen Augen als diffuses Licht empfunden wird. Unter diesen Bedingungen ist es mir jedoch sehr viel schwerer, zu entscheiden, welches Auge lichtempfindend ist. Käme es nur auf das Abblendungsgefühl an, so sollte vielleicht unter diesen Bedingungen an ehesten der Entscheid möglich sein. Der Versuch spricht nicht dafür, dass das periphere Gesichtsfeld in meiner früheren Versuchsanordnung wesentich mitbeteiligt gewesen ist und für die Onterscheidbarkeit rechts- und linksäugiger Eindrücke einen Anhaltspunkt geboten hat."

Offenbar fasst Heine hier zwei Momente, welche wir stets streng geschieden haben, in eins zusammen: nämlich die periphere

1) Pflüg er's Arch. Bd. 90 S. 292.

2) Ebenda S. 301. 
Erhellung und das Abblendungsgefühl. Zunächst aber handelt es sich hier aber noch nicht um das letztere.

Nebenbei möchten wir bemerken, dass es nicht verwunderlich ist, wenn das Abblendungsgefühl bei geschlossenen Augen, wie Heine es bei seiner Versuchsanordnung auch angibt, hier nicht eintritt, denn das scheinbare Organgefühl äussert sich ja sehr bäufig darin, dass der Beobachter die Empfindung leichten Lidschlusses auf dem verdunkelten Auge hat. Es stände eher zu erwarten, dass ein Gefühl des Offenseins des belichteten, in Wirklichkeit geschlossenen Auges einträte, etwa in der Art, wie wir das in unserer zweiten Arbeit ${ }^{1}$ ) bei Versuchen mit monokularen Nachbildern beschrieben haben. In der Tat glauben wir bei der Wiederholung des oben beschriebenen Heine'schen Versuches bei geschlossenen Augen, wenn überhaupt etwas, dann das Gefühl des Offenseins des belichteten Auges zu empfinden. -

Wir wollen noch mit wenigen Worten auf den Gedanken Hein e's ${ }^{2}$ ) eingehen, dass es sich bei dem Abblendungsgefühl um ein binokulares Kontrastphänomen handeln könnte. Wir glauben, dass diese Auffassung sich nicht als zutreffend erweisen wird, weil der binokulare optische Kontrast eben rein optisch ist und sich nicht als ein scheinbares Organgefühl äussern kann. Wir verweisen auch hier auf die soeben besprochene Empfindung des Offenseins eines tatsächlich geschlossenen Auges. Ob nun diese Organempfindungen mit dem binokularen Kontrast irgendwie zusammenhängen, bedarf wohl genauerer Untersuchung, wie Heine ja auch selbst angibt. Uns scheint ein derartiger Zusammenhang nicht wahrscheinlich, da wir das Abblendungsgefühl ausser bei Differenzen in der Lichtintensität der optischen Reize beider Augen ebenfalls, wenn auch in schwächerem Masse, bei geringerem Konturenreichtum und geringerer Schärfe des Bildes eines Auges beobachten konnten. Von einem monokularen oder binokularen Kontrast hinsichtlich Schärfe oder Konturenreichtum der optischen Bilder ist aber unseres Wissens bisher nichts bekannt. Das Überwiegen der Konturen bei binokularem Wettstreit wird man kaum geneigt sein, als Kontrasterscheinung aufzufassen. -

Wir wollen zum Schlusse noeh kurz der Frage gedenken, wie es sich im Hinblick auf die festgestellten Beobachtungstatsachen mit

1) Pflüger's Arch. Bd. 91 S. 368 f.

2) Pflüger's Arch. Bd. 101 S. 68. 
den Prüfungsmethoden auf Simulation einseitiger Blindheit und Schwachsichtigkeit verhält. Heine hatte diesen Punkt in seiner ersten Arbeit gar nicht berührt, und zwar, wie er in seiner letzten Veröffentlichung sagt, aus dem Grunde, weil ihn die ganze Frage der Unterscheidbarkeit überhaupt nur theoretisch interessiert habe; er sei auf eine Besprechung der Simulationsproben, welche auf der Unmöglichkeit einer Unterscheidbarkeit beruhen, absichtlich nicht eingegangen, da hier stets beide Augen ziemlich ähnliche Bilder sähen, eine Unterscheidung in dem von ihm angegebenen Sinne also gar nicht erwartet werden könne. Wir sind nun der Ansicht, dass, wenn Heine auch vorzugsweise Versuche mitgeteilt hatte, bei welchen die Bilder beider Augen einander mehr oder weniger unähnlich waren, in Heine's Versuch 8 eine grosse Ähnlichkeit in dieser Beziehung vorhanden gewesen ist. Heine beschreibt denselben mit folgenden Worten ${ }^{1}$ ):

„Einen ähnlichen Versuch kann man am Hering'schen Haploskop anstellen: Bietet man dem linken Auge einen gelbroten Fleck von etwa $2-3 \mathrm{~cm}$ Durchmesser, dem rechten einen ebensolchen gelbgrünen, so sehen wir entweder. nur den einen oder den anderen und wissen dann genau, mit welchem Auge wir ihn sehen, oder aber wir sehen eine Mischfarbe, und dann wissen wir natürlich nicht, mit welchem Auge wir sehen, d. $h$. wir sehen dann mit beiden Augen. Je ausgesprochener der Wettstreit ist, je mehr das eine Auge darin unterliegt, um so leichter ist zu sagen, welches von beiden das sehende ist."

Es handelt sich also hier um (abgesehen von der Farbe) objektiv gleichwertige Bilder, wie auch bei den meisten binokularen Simulationsproben. Abweichend von den letzteren ist freilich nur der Umstand, dass bei diesen die eventuell zu kombinierenden Einzelbilder nicht auf korrespondierenden Netzhautstellen sich abbilden, wie das beim $\mathrm{He}$ in e'schen Versuch der Fall ist. Immerhin schien uns der alleinige Hinweis Heine's auf die Bedeutungslosigkeit für das körperliche Sehen nicht präzise genug, um die Bedenken, welche jener Versuch zusammen mit den theoretischen Sätzen Heine's zu erwecken geeignet war, zu zerstreuen, ganz abgesehen davon, dass körperliches Sehen noch nicht binokulares Sehen überhaupt ist. Es erscheint uns darum auch nicht so ungerechtfertigt wie $\mathrm{H}$ eine, wenn wir in unserer ersten Arbeit diesen Punkt berührt haben, ohne freilich näher auf denselben einzugehen.

1) Klin. Monatsbl. f. Augenheilk. I. c. S. 619. 
Nochm. zur Frage d. Unterscheidbarkeit rechts- u. linksäugiger Eindrücke. 289

Denn wir waren im Gegensatz zu Heine bei Wiederholung seines oben zitierten Versuches richt imstande zu unterscheiden, welches Auge beim Wettstreit den Sieg davon trug. Wir betonten darum auch an anderer Stelle mit Bezug auf das Abblendungsgefühl, dass die subjektive Unterdrückung eines Bildes von zwei im erwähnten Sinne gleichwertigen Bildern nicht imstande ist, die Organempfindung auszulösen ${ }^{1}$ ). Auch neuerdings haben wir unsere Beobachtung wieder bestätigen können.

Es können also die Simulationsproben, in welchen es sich um Eindrücke etwa gleichen Wertes in beiden Augen handelt, als einwandsfrei bezeichnet werden; dagegen ergeben sich einige Bedenken gegen diejenigen Methoden, bei welchen eine erhebliche Ungleichwertigkeit der optischen Bilder, z. B. durch Vorsetzen von starken Konvex- bezw. Konkavgläsern vor das angeblich allein gute Auge erzeugt, oder bei denen durch entsprechend gefärbte Gläser das eine Auge vom Sehakte ausgeschlossen wird. In derartigen Fällen muss es nicht als unmöglich angesehen werden, dass der Simulant durch die Entstehung des Abblendungsgefühls darauf aufmerksam gemacht wird, dass mit seinem "guten" Auge irgend etwas nicht in Ordnung ist. Wenn also auch diese Methoden sehr oft positive Resultate geben, so kann umgekehrt ein negativer Ausfall des Versuchs der Entlarvung mittels derselben noch nicht unbedingt als ein Beweis für die Schwachsichtigkeit bezw. Blindheit des einen Auges angesehen werden, ein Umstand, der ja dem routinierten Augenarzte bekannt sein dürfte.

1) Pflüger's Arch. Bd. 91 S. $363 \mathrm{f}$. 